\section{Rotational Relaxation of Free and Solvated Rotors}

A.J. Bain, C. Han, P.L. Holt, P.J. McCarthy, A.B. Myers, M.A. Pereira, and R.M. Hochstrasser

Department of Chemistry, University of Pennsylvania,

Philadelphia, PA 19104, USA

\section{Introduction}

In mast experinental studies of nolecular motion in liquids the signals ar averaged orer nany collisional pericals and the pocesses observed are diffusive. When stuaies are carried out with sufficiently short light pulses it ahould becoma possible to observe nondiffusive behavior. In the case of molosular recrientation dynamics the nondiffubive portion corresponds to nesrly free rotation. It is of great interost to study these transient regines because of the detalled inforration that can be obtalned about colli sicn dynanics in condensed phases. While these tranticies are well known through atudies of Rayleigh scattering $[1]$, lnired, and [2] and fram time donaln kerr creet stuales in neat ing the electronically

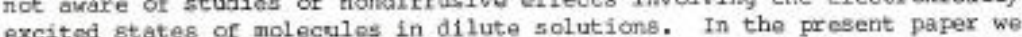
a1scuss fluorescence and polarization spectroscopy as methods of approach and resent prelininary results which bring us claser to realizing these goals.

cor a short period after vitrashort pulse excitation a molecule in a solution can be cansidered to be freely rotating lnasmach as it will have detinite values of the angular nonientum, J, and its projections chto laboratory and molecule-fixed axes. The collisions will cause the Initial J and lts projections to randonize [5]. The initial situation correspons clafofo that which would prevail in a cas at the same temperature. We therefore began our investigations with studies of yases totry to rapid conpared with collisions the nondiffusive behavior should be dominant whereas in the other ligit the diffusional regime should be reached before the molecule can roate signifieantly and the nondiffusive effect will be vanishingly small.

\section{Fluorescence and Polarization Methods}

In a fluorescence experinent the sample is excited with a short pulse of linearly polarized light and the tims evelution of the fluorescence aniso tropy, $r(t)=\left(I_{d}-I_{1}\right) /\left(I_{n}+2 I_{1}\right)$, is measured, In tine-resolved polarization apectroscopy the decay of the anisotrogy induced by a Dolarized excitation pulse is measured by probing with a secand pulse polarized at $45^{\circ}$ to the first and detecting the intensity transmitted through orossed polarizers. In solution, if the recorientation can be described as rotational diffusion, $r(t)$ decays as a sum of exponentials and the polarization spectrcocopy sig-

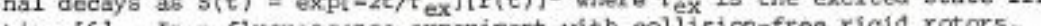

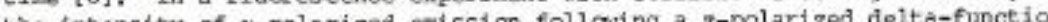
excitation pulse 15 given by [7]

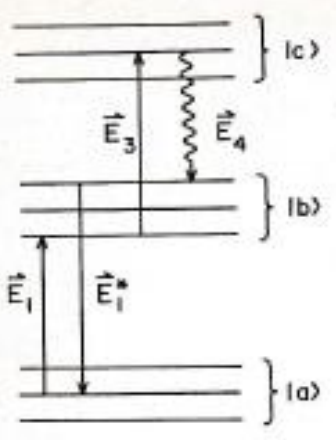

19. I Energy level diagram describing polarization spectroecopy and fluores-

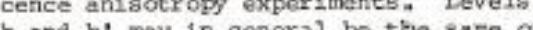
atererent states: In flumescence, Is belou $b$ and $b^{\prime}$ in enercy and the $b \rightarrow c$ and $b^{\prime} \rightarrow c$ processes reprosent spontancous emission.

$$
I_{z \alpha \alpha}(t) \propto \sum_{a, c} P_{a}\left|\sum_{b} e^{-1 \Phi_{b} t} c_{\alpha}^{c b} c_{z}^{b a}\right|^{2},
$$

where $a, b$, and $c$ are the initial, intermediate, and final rotational state (see Fig. 1), $P_{a}$ is the initial population of state $a$, and $\mathrm{c}_{\mathrm{g}}^{\mathrm{c}}=\left\langle\mathrm{c}\left|\mathrm{C}_{\mathrm{a}}\right| \mathrm{b}\right\rangle$ to the direeticn cosine matrix element between the dipole axis of the mole-fixed axis $\alpha$. The vibraticnal states factor out of the aniscopy signal is given by

$$
S(T) \propto \int_{T}^{\infty} d t\left[\hat{P}^{(3)}(t) \cdot \hat{e}_{4}\right]^{2},
$$

where $T$ is the delay time between pump and probe pulses, and the macroscoptc polarization $\vec{f}(3)(t)$ is given for free $x$ igid rotors by [7]

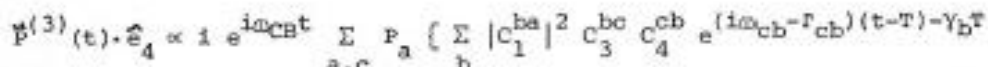

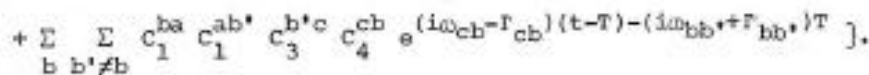

The pump, probe, and detected fields have polarizations $\hat{e}_{1}, \hat{e}_{3}$, and $\hat{e}_{4},{ }^{\mathrm{r}} \mathrm{cb}$ is the total dephasing constant between the final pair of levele, $\gamma_{b}$ and $\mathrm{T}_{\mathrm{hb}}$. are the population decay and dephasing consta

For collision-free molecules $r(t)$ does not in general decay to zero because the total angular monentum is conserved. For an ensemble of regular rotors at high J, there is a transient near time zero which decays to a constant value on a time Ecale of n(1/KT) This transient, which represents $x$ 1gid-body rotational motion in a elassical picture, arises in a quantum ntechanical treatment frcm interferences between Aifferent intermediate rctaticnal states that are doupled to the sa pair of initial and final states [7] iFlg. 11. The anisotrops from a regces, but the beating between different finitiol J's cancels all bat ane of these recurrences, learing a constant lang-time amisotrogy for the 15olated molecule that dopende on the inertial ratice $[8,9]$. Collisions will cavee the long-time anisotrogy to apreach zero, bat even at high collision rates 
(i.e., in solution) the decay at very short times should resemble that for the free rotar,

The polarization spectroscopy signal for free rotors depends not only on the inertial ratios but also on the dephasing rate between the final pairs of coupled levels because this damps the macroscopic polarization in the medium. If dephasing is much faster than rotational periods, the polarization and fluorescence anisotropg decays axe related by $s(t)=\exp \left[-2 t / \tau_{\mathrm{ex}}\right]$ $[r(t)]^{2}$ as for rotational diffusion. If dephasing is slow compared with rotation, a different polarization decay is expected which bears no simple relaticnshlp to the fluar esconce anisotropy (see Fig. 2 ). In adaltion there the second-order density mat-

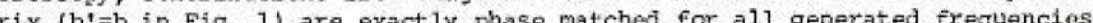
hisle off-alagon l cantributions (rotational CSPS processes) are not. The lile off-diagonal (oscillatory) terms thus depend on the spectral widths of the pulses relative to the spacing between $P, Q$, and $R$ branches, the interaction length, and the angle between pusmp and probe beams (7). Finally, even in the absence of vihration-rotation interaction, excitation of more than one intermediate yibrational state causes the nonlinear signal to decay at a rate determined by the energy spread of the coupled vibrational levels.

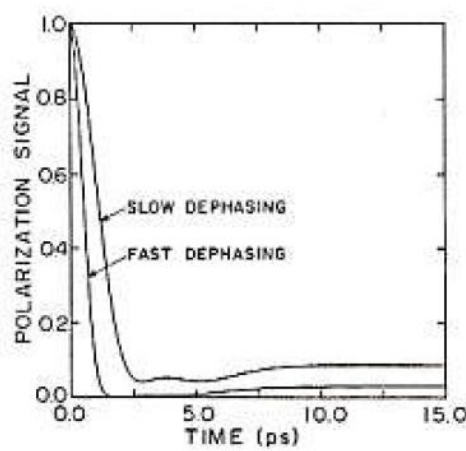

Fig, 2 Calculated polarization spectroscopy decays for fluorene thodeled as a symetric top in the fast and slow dephasing limits (see text). Rotational constants wexp $\mathrm{A}=0.074 \mathrm{~cm}^{-1}$ and $\mathrm{B}=0.0175$ $\mathrm{cm}^{-1}$, at $\mathrm{T}=443 \mathrm{~K}$. Delta-functic pulses were assumed. Signal strengths are not to scale; the signal is much stronger in the slow dephasing limit.

\section{Results and Discussion}

We have recently observed a free rotation transient in the fluorescence anisotropy of stilbene vapor $(2 \mathrm{gg}$. 3) [10]. An overall time resolution of 4-5 p5 was achievo by upeonverting the uv flucrescence in potassium pentaborate the light 504 nm light at 604 nta was used to gate the fluorescence. I $I_{l l}$ and $I_{1}$ were obtain on successive scans by rotating the polarization of the excitaticn pulse fluorescence. Both the observed zero-time anisotropy of 0.16 and the general shape of the anisotropy decay are reproduced quite well by convoluting the theoretical regular rotor decay with a 5 ps instrument function. The observed long-time anisotropy of $0.069 \pm 0.003$ lies between the value of 0.074 expected for a regular rotor with stilbene's inertial ratios [9] and the value of 0.056 expected for fully statistical rotation in which extensive vibrationrotation coupling genexates a microcanonical distribution of $\mathrm{K}$ levels for

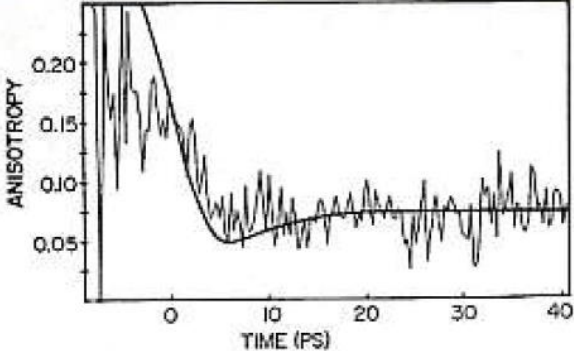

Fig. 3 Fluorescence anisotropy decay of trans-stilbene vapor at $463 \mathrm{~K}$, 1 torr, with $302 \mathrm{~nm}$ excitation. The smooth curve is the theoretical decay for a symmetric top with $\mathrm{A}=0.0908 \mathrm{~cm}^{-1}$ and $\mathrm{B}=0,00 \mathrm{~B} 65 \mathrm{~cm}^{-1}$. A Gaussian instrument function of 5 ps Fwhy was usec.

each I state [8]. This indicates that the hot $(463 \mathrm{~K})$ stilbene molecule excited $650 \mathrm{~cm}^{-1}$ above the origin undergoes partial but incomplete vibration-

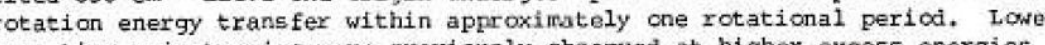
(11); above the origin correspends to essentially statistical rotation.

Figure 4 shows the polarization spectroscopy response of fluorene vapar. Excitation and probe pulses (295 $\mathrm{nm}, 4 \mathrm{ps}$ and $592 \mathrm{~nm}, 6 \mathrm{nS}$ ) were derived from an excimer amplified synchronously pumped dye laser system [12]. The constant signal level observed fron a few ps to 250 ps indicates the absence of any vibration-rotation coupling that could destroy the anisotropy on this time scale. Pluorescence experiments [13] yielded a value of 0.066 for the anisotropy of fluorene excited at its 0-0 transition, indicating that it behaves as a regular rotcr. We have determined that the small spike at zero time in the polarization experiment originates from the cell windows. No free rotation transient due to the vapor can be discerned. However, this is consistent with calculations in which the appropriate pulse wiatis and phase that are closer to the $1.7 \mathrm{ps}$ free rotation time of fluorene.

In addition to the vapor phase work, we have obtained fluorescence anisoIn adation to the kapor in low and high viscosity solvents [10]. The decays tropy decays for stilbene in low and high viscosity solvents [10]. The decays

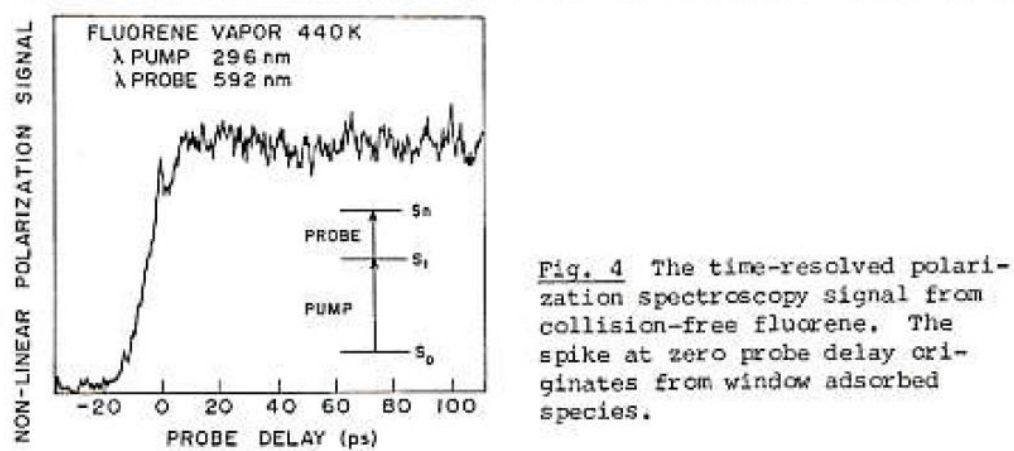

492 
in isopentane and hexadecane, respectively, with corresponding zero-time anisotropies of 0.39 and 0.36 . No nondiffusive behavior, which would be nanifested as a rapid decrease in the anisotropy near $t=0$, is apparent.

Motions such as the in-plane spinning of benzene and substituted benzenes require little solvent displacement and appear more likely to exhibit reor lentation by free rotation. Previcus investigations using BMR and light much shorter than predict independent of viscosity [1]. We have recently obtained fluorescence aniso tropy decays for a substituted benzene, aniline, to directly measure these orientational correlation functions (Fig. 5). Fitting the data to single exponential decays ylelds rotational relaxation times that differ by only factor of two between isopontane $\{\eta=0.22 \mathrm{cp})$ and hexadecane $(\eta=3,3\}$. The electronic transition is b-axis polarized (in the plane of the ring perpendicular to the $\mathrm{CN}$ bond) so the fluorescenco can depolarize by rotation about both the a and $c$ axes. "Slip" bountary conditions [14] predict rotational diffusion times of $\tau_{a}=0.7 \mathrm{ps}, \tau_{c}=0.1 \mathrm{ps}$ in isopentane and $\tau_{\mathrm{a}}=10 \mathrm{ps}, \tau_{\mathrm{c}}=1.6 \mathrm{ps}$ in hexadecane. The $\tau_{\mathrm{c}}$ values are small because aniline 15 a near-oblate ellipsoid and rotation about the symnetry axis of a synmetric top experioncos no friction in the slip limit. Presumably the above rotational diffusion thes should be adaed to the free rotation times (the expected zero-viscosity intercepts) of $\tau_{a}=0.4 \mathrm{ps}, \tau_{c}=0.7$ ps. It is difficult to determine how well exponentials to the anisotropy decays and because sitive to the axial ratios used [14]. Horever, ${ }^{2}$, in particular, is senall rearientation time is within a factor of five of the free rotation time.

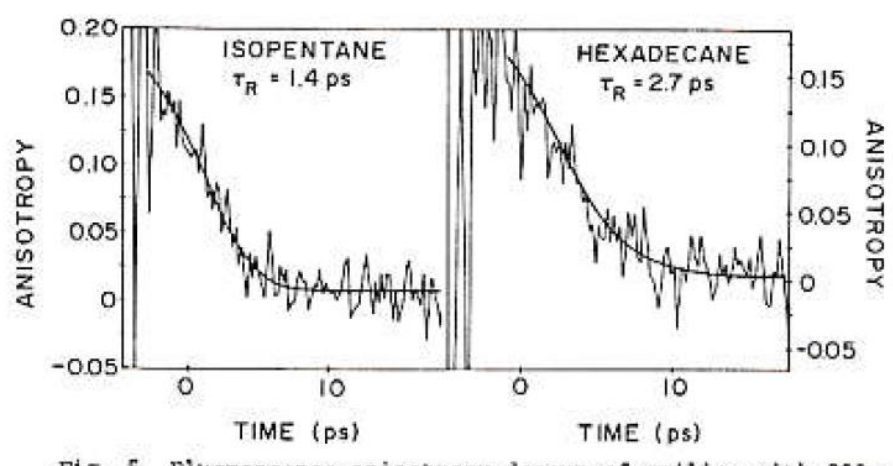

Fig. 5 Fluorescence anisotropy decays of aniline with $302 \mathrm{~nm}$ excitation, The smooth curves are the best fits to single exponential decays.

\section{Conclusions}

We have observed a transient in the fluorescence anisotropy of stilbene vapo that can clearly be attributed to free inertial rotation. In contrast, polarization spectroscopy did not resolve any such transient in fluorene vapor. contribute to the four transient less apgarnt While these facs and could render the free rotaticn four-wave mixing data, they can also provide additional information, Por doctoral fellow. example, by changing the interaction length or the angle between pump and probe beams it may be possible to experimentally separate the diagonal and off-diagonal contributions to the polarization signal. Comparison of polarization spectroscopy and fluorescence anisotropy decays may also allow determination of the dephasing constant $\mathrm{r}_{\mathrm{bc}}$ in (3). It should now be feasible to apply fluorescence methods to examine the extent or free rotation of medium sized molecules in liquids, dense gases, and supercritical fluids. preliminary results suggest that free rotation may contribute considerably to reorientation of aniline in solution. Such studies will provide an important link between previous work on rotational diffusion in larger nolecules performed with lower

\section{Acknowledgments}

This work was supported by grants from NSF and NIH. A.B.M. is an NIH post-

\section{References}

1. B. J. Berne and R. Pecora: Dynamic Light Scattering (Wiley, New York 1976), chapter 7

2. J. H. R. Clarke: In Advances in Infrared and Raman Spectroscopy, ed. by R. J. H. Clark and R. E. Hester, vol. 4 (Heyden, London 1978)

4. B. I. Greene and R. C. Farrow: In Picosecond Phenomena III, ed. by K. B. Eisenthal, R. M. Hochstrasser, w. Kaiser, and A. Laubereau (SpringerVerlag, Berlin, Heidelberg 1982)

5. R, G, Gordon: J. Chem, Phys, 44, 1830 (1966)

6. A. B. Myers and R. M. Hochstrasser: IEEE J. Quantum Electron., in press 7. A. B. Myers and R. M. Hochstrasser: J. Chem. Phys., submitted

8. G. M. Nathanson and G. M. McClelland: J. Chem. Phys. 81, 629 (1984)

9. A, P, Blokhin and V. A. Tolkachev: Opt. Spectrosc. 51 , 152 (1981)

10. A. B. Myers, P. L. Holt, M. A. Pereira, and R. M. Hochstrasser: Chem. Phys. Lett., submitted

11. D. K. Negus, D. S. Green, and R. M. Hochstrasser: Chem. Phys. Lett. 117, 409 (1985)

12. A. J. Bain, P. J. McCarthy, and R. M. Hochstrasser: Chem. Phys. Lett. 125,307 (1986)

B. Mochstrasser: Unpublished results

14. G, K, Youngren and A. Acrivos: J, Chem. Phys. 63, 3846 (1975) 